\title{
Assessment of Artificial Neural Networks for Hourly Solar Radiation Prediction
}

\author{
Tamer Khatib, ${ }^{1}$ Azah Mohamed, ${ }^{1}$ K. Sopian, ${ }^{2}$ and M. Mahmoud ${ }^{3}$ \\ ${ }^{1}$ Department of Electrical, Electronic and System Engineering, Faculty of Engineering and Built Environment, \\ Universiti Kebangsaan Malaysia, Selangor, Bangi 43600, Malaysia \\ ${ }^{2}$ Solar Energy Research Institute, Universiti Kebangsaan Malaysia, Selangor, Bangi 43600, Malaysia \\ ${ }^{3}$ Department of Electrical Engineering, Engineering Faculty, An-Najah National University, Nablus 97300, Palestine
}

Correspondence should be addressed to Tamer Khatib, tamer_khat@hotmail.com

Received 14 January 2012; Accepted 24 February 2012

Academic Editor: Jincai Zhao

Copyright ( $) 2012$ Tamer Khatib et al. This is an open access article distributed under the Creative Commons Attribution License, which permits unrestricted use, distribution, and reproduction in any medium, provided the original work is properly cited.

This paper presents an assessment for the artificial neural network (ANN) based approach for hourly solar radiation prediction. The Four ANNs topologies were used including a generalized (GRNN), a feed-forward backpropagation (FFNN), a cascadeforward backpropagation (CFNN), and an Elman backpropagation (ELMNN). The three statistical values used to evaluate the efficacy of the neural networks were mean absolute percentage error (MAPE), mean bias error (MBE) and root mean square error (RMSE). Prediction results show that the GRNN exceeds the other proposed methods. The average values of the MAPE, MBE and RMSE using GRNN were 4.9\%, 0.29\% and 5.75\%, respectively. FFNN and CFNN efficacies were acceptable in general, but their predictive value was degraded in poor solar radiation conditions. The average values of the MAPE, MBE and RMSE using the FFNN were $23 \%,-.09 \%$ and $21.9 \%$, respectively, while the average values of the MAPE, MBE and RMSE using CFNN were $22.5 \%$, $-19.15 \%$ and $21.9 \%$, respectively. ELMNN fared the worst among the proposed methods in predicting hourly solar radiation with average MABE, MBE and RMSE values of $34.5 \%,-11.1 \%$ and $34.35 \%$. The use of the GRNN to predict solar radiation in all climate conditions yielded results that were highly accurate and efficient.

\section{Introduction}

Solar radiation is the part of the Sun's radiation which falls at the Earth's surface. This energy is available for many applications, such as increasing water's temperature or moving electrons in a photovoltaic cell. Moreover, it supplies energy to natural processes like photosynthesis. Solar energy is secure, clean, and available on the Earth throughout the year. Its secure and clean applications are very important to the world, especially at a time of fossil fuel high costs and the critical situation of the atmosphere resulting from fossil fuel applications. Solar radiation data provides information on how much is the Sun potential at a location on the Earth during a specific time period. These data are very important for designing sizing solar energy systems. Due to the high cost and installation difficulties of measurement, these data are not always available. Therefore, there is a demand to develop alternative ways of predicting these data.
A solar radiation database is very important part of an energy efficiency policy [1].

In recent years, ANNs have been used in solar radiation modeling for locations at different latitudes and with different climates using ANN. Related work has been done in countries such as Saudi Arabia, Oman, Spain, Turkey, China, Egypt, Cyprus, Greece, India, Algeria, and the UK [2-10]. In these works the authors used the feedback propagation neural network, while better ANN networks may be more accurate in predicting solar radiation.

However, solar prediction methods that have been developed for Malaysia are found in [11, 12]. In [11], the solar radiation data for three locations were considered without using any prediction algorithms. In [12], an algorithm was developed for predicting the monthly solar radiation based on the least squares linear regression analysis using data from eight locations. To provide a comprehensive database for the solar energy potential in Malaysia, an ANN model is 
proposed to allow prediction of hourly solar radiation levels. The main objective of this research is to propose an assessment for ANN-based approach for solar radiation prediction in order to develop accurate models for predicting hourly solar radiation for Kuala Lumpur based on the number of sunshine hours, day, month, temperature, humidity, and location coordinates. The accuracy of the four ANN models will then be compared to determine the most accurate ANN model for predicting the hourly solar radiation. In this work, long-term data on solar irradiation levels (1975-2005) taken for Kuala Lumpur were used. These data were provided by the Solar Energy Research Institute of Universiti Kebangsaan Malaysia (UKM).

\section{Solar Radiation Components}

Solar radiation is classified into two main categories: extraterrestrial solar radiation $\left(G_{\text {extra }}\right)$ and global solar radiation $\left(G_{T}\right) . G_{\text {extra }}$ represents the total solar radiation above the atmosphere while $G_{T}$ is the total solar radiation under the atmosphere. Then $G_{\text {extra }}$ is given by

$$
G_{\text {extra }}=I_{o}\left[1+.034 \cos \left(\frac{2 \pi N}{365}\right)\right]
$$

where $I_{o}$ is the solar constant equal to $1367 \mathrm{~W} / \mathrm{m}^{2} . N$ is the number of the day (i.e. $01 \mathrm{FEB}=32$ ).

The global solar irradiation $\left(E_{T}\right)$ on a tilted surface consists of three components:

$$
G_{T}=G_{B}+G_{D}+G_{R}
$$

where $G_{B}, G_{D}$, and $G_{R}$ are the beam (direct), diffused, and reflected solar radiations. On a horizontal surface, $G_{R}$ is equal to zero; therefore, $G_{T}$ on a horizontal surface is given by

$$
G_{T}=G_{B}+G_{D}
$$

Global radiation $\left(G_{T}\right)$ can be calculated using $G_{\text {extra }}$ as shown below:

$$
\frac{G_{T}}{G_{\text {extra }}}=K_{T}
$$

where $K_{T}$ is the sky clearness index. Diffused radiation can be calculated by

$$
\frac{G_{D}}{G_{\text {extra }}}=K_{D}
$$

where $K_{D}$ is the diffuse index.

\section{Artificial Neural Network for Hourly Solar Radiation Prediction}

Artificial neural networks, ANNs, are nonalgorithmic and intensely parallel information processing systems. They learn the relationship between the input and output variables by mastering previously recorded data. An ANN consisted of parallel elemental units called neurons. The neurons are connected by a large number of weighted links which

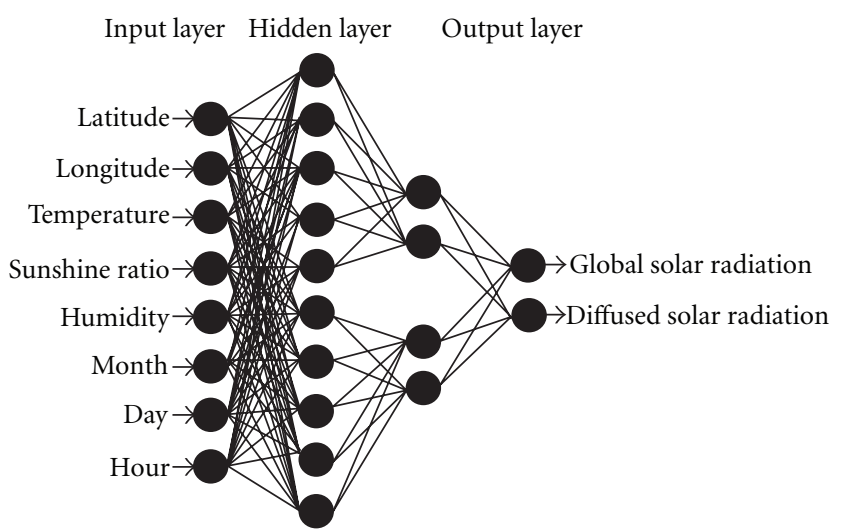

FIGURE 1: GRNN for solar radiation prediction.

passes signals or information. Simply, a neuron receives and combines inputs and then generates the final results in a nonlinear operation. The term ANN usually refers to a multilayer perceptron (MLP) network. However, there are many other types of neural networks, including probabilistic neural networks (PNN), general regression neural networks (GRNN), radial basis function networks (RBF), cascade correlation, functional link networks, Kohonen networks, Gram-Charlier networks, learning vector quantization, Hebb networks, Adaline networks, heteroassociative networks, recurrent networks, and hybrid networks [13].

In this study, the four networks used to predict hourly solar radiation for Kuala Lumpur are the generalized regression neural network (GRNN), the feed-forward back propagation neural network (FFNN), the cascade-forward back propagation neural network (CFNN), and the Elman back propagation neural network (ELMNN). These networks were selected based on the fact that they are among the most commonly used neural networks [6, 13] MATLAB was used to train and develop the ANN models to predict hourly solar radiation. Eight geographical and climatic variables were used as input parameters including hour, day, month, latitude, longitude, temperature, humidity, and daily sunshine hours ratio (i.e., measured sunshine duration over daily maximum possible sunshine duration). The two nodes in the output layer were estimated hourly global and diffused solar radiations.

3.1. Generalized Regression Neural Network. The generalized regression neural network (GRNN) is a probabilistic-based network. This network makes classification where the target variable is definite, while GRNNs make regression where the target variable is continuous. Figure 1 shows the GRNN diagram for hourly solar radiation prediction.

The network consists of input, hidden, and output layers. The input layer has one neuron for each predictor variable. The input neurons standardize the range of values by subtracting the median and dividing by the inter quartile range. The input neurons then feed the values to each of the neurons in the hidden layer. In the hidden layer, there is one neuron for each case in the training data set. The neuron stores the 


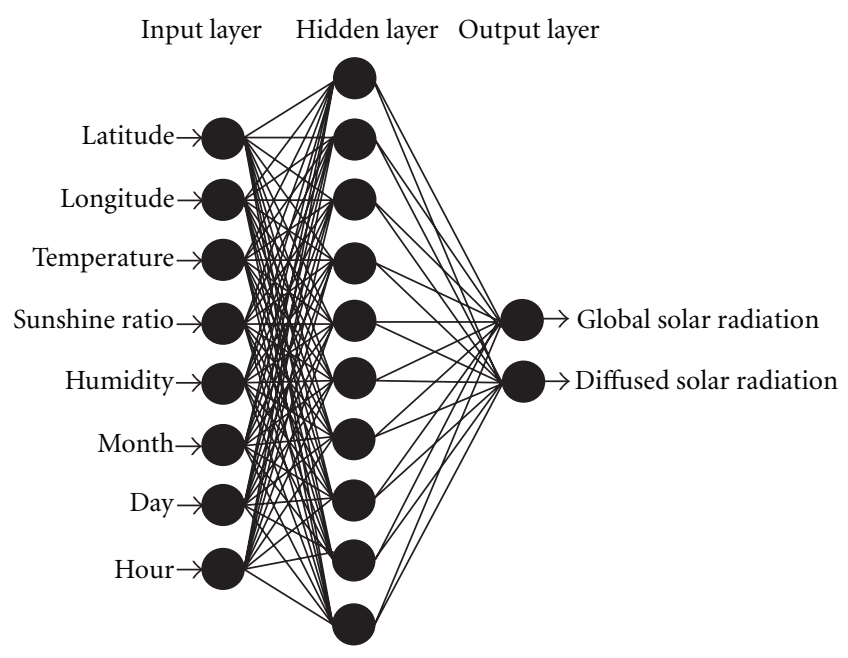

FIGURE 2: FFNN for solar radiation prediction.

values of the predictor variables for each case along with the target value. When presented with the vector of input values from the input layer, a hidden neuron computes the Euclidean distance of the test case from the neuron's center point and then applies the RBF kernel function using the sigma value(s). The resulting value is passed to the neurons in the pattern layer. However, the pattern layer (summation layer) has two neurons: one is the denominator summation unit and the other is the numerator summation unit. The denominator summation unit adds up the weights of the values coming from each of the hidden neurons. Meanwhile, the numerator summation unit adds up the weights of the values multiplied by the actual target value for each hidden neuron. Finally, the decision layer divides the value accumulated in the numerator summation unit by the value in the denominator summation unit and uses the result as the predicted target value $[13,14]$.

3.2. Feed-Forward Backpropagation Network. Figure 2 shows the feed-forward back propagation (FF) network diagram for solar radiation prediction. However, feed forward means that the values only move from the input layer to the hidden layer and then to the output layers.

FFNN, a type of multilayer perceptron network (MLP), has an input layer which standardizes these values so that the range of each variable is -1 to 1 . The input layer distributes these values to each of the neurons in the hidden layer. In addition to the predictor variables, there is a constant input of 1.0, called "the bias," that is fed to each of the hidden layers. The bias is multiplied by a weight and added to the sum going into the neuron. The hidden layer represents the phase where the value from each input neuron is multiplied by a weight, and the resulting weighted values are added together producing a combined value. The weighted sum is fed into a transfer function, which then outputs a value. The outputs from the hidden layer are then distributed to the output layer. The output layer is the final layer where the value from each hidden layer neuron is multiplied by a

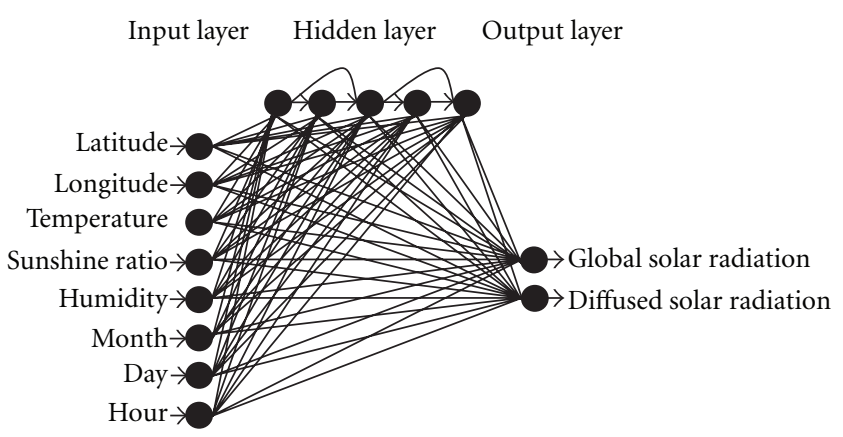

FIGURE 3: CFNN for solar radiation prediction.

weight, and the resulting weighted values are added together producing a final combined value. The weighted sum is fed into a transfer function, which then yields the outputs of the network $[13,14]$.

3.3. Cascade-Forward Backpropagation Network. Cascade correlation neural networks are "self-organizing" networks. The network begins with only input and output neurons. It is called a cascade because the output from all of the neurons is already in the network that feeds into new neurons. As new neurons are added to the hidden layer, the learning algorithm attempts to maximize the magnitude of the correlation between the new neuron's output and the residual error of the network that we are trying to minimize. A cascade neural network has three layers: input, hidden, and output. The input layer is a vector of predictor variable values. The input neurons do not perform any action on the values other than distributing them to the neurons in the hidden and output layers. In addition to the predictor variables, there is a constant input of 1.0, called the bias that is fed into each of the hidden and output neurons; the bias is multiplied by a weight and added to the sum going into the neuron. In the hidden layer, each input neuron is multiplied by a weight, and the resulting weighted values are added together to produce a combined value. The weighted sum is fed into a transfer function, which then outputs a value. The outputs from the hidden layer are distributed to the output layer that receives values from all of the input neurons (including the bias) and all of the hidden layer neurons. Each value presented to an output neuron is multiplied by a weight, and the resulting weighted values are added together again to produce a combined value. The weighted sum is fed into a transfer function, which then outputs the final value $[13,14]$. Figure 3 shows the CFNN diagram for solar radiation prediction.

3.4. Elman Back Propagation Network. Elman networks are a form of recurrent neural networks that have connections from their hidden layer back to a special copy layer. This means that the function learned by the network can be based on the current inputs plus a record of the previous state(s) and outputs of the network. In other words, the Elman net is a finite state machine that learns what state to remember (i.e. what is relevant). The special copy layer is 
treated as another set of inputs so standard backpropagation learning techniques can be used $[13,14]$. Figure 4 shows ELM network for solar radiation prediction.

3.5. Artificial Neural Networks Evaluation Criteria. To evaluate the proposed neural networks, three error statistics were used. These statistics are mean absolute percentage error (MAPE), mean bias error (MBE), and root mean square error (RMSE). MAPE is an indicator of accuracy. It usually expresses accuracy as a percentage and is defined by the following formula:

$$
\mathrm{MAPE}=\frac{1}{n} \sum_{t=1}^{n} \frac{I-I_{p}}{I},
$$

where $I$ is the real value and $I_{p}$ is the predicted value. The difference between $I$ and $I_{p}$ is divided by $I$. The resultant of this calculation is summed for every fitted or forecasted point in time and divided again by the number of fitted points, $n$. This formula makes it a percentage error so one can compare the error of fitted time series that differ in level.

In addition, most ANN models are being evaluated using MBE and RMSE. MBE is an indicator for the average deviation of the predicted values from the measured data. A positive MBE value indicates the amount of overestimation in the predicted global solar radiation and vice versa. On the other hand, RMSE provides information on the short-term performance of the models and is a measure of the variation of the predicted values around the measured data. It indicates the scattering of data around linear lines. Moreover, RMSE shows the efficiency of the developed network in predicting future individual values. A large positive RMSE implies a big deviation in the predicted value from the measured value. $\mathrm{MBE}$ and RMSE are defined as follows:

$$
\begin{aligned}
\mathrm{MBE} & =\frac{1}{n} \sum_{i=1}^{n}\left(I_{P_{i}}-I_{i}\right), \\
\mathrm{RMSE} & =\sqrt{\frac{1}{n} \sum_{i=1}^{n}\left(I_{P_{i}}-I_{i}\right)^{2},}
\end{aligned}
$$

where $I_{P i}$ is the predicted value, $I_{i}$ is the measured value, and $n$ is the number of observations.

\section{Results and Discussion}

The proposed networks were trained using $80 \%$ of the provided data, while the remaining $20 \%$ was used to test the trained network. Random solar radiation profiles for September 2000, March 2002, and June 2004 were used to calculate the results. The proposed ANNs predicted the solar radiation values (global and diffuse), and then the predicted results were compared with the measured data. Each neural network was tested individually using the hourly solar radiation values, and all of the proposed ANNs were compared together using the monthly average solar radiation values.

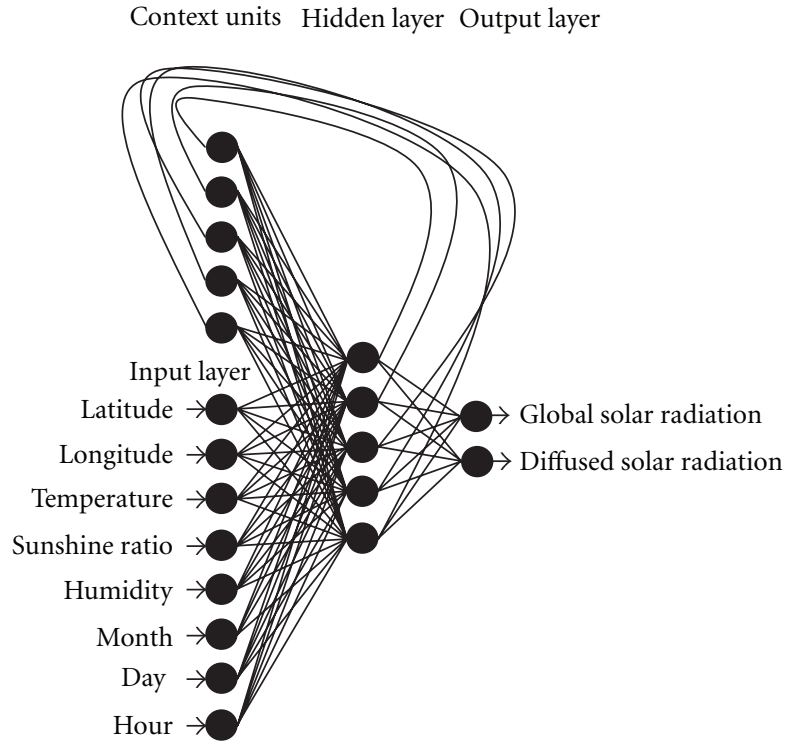

FIGURE 4: ELMNN for solar radiation prediction.

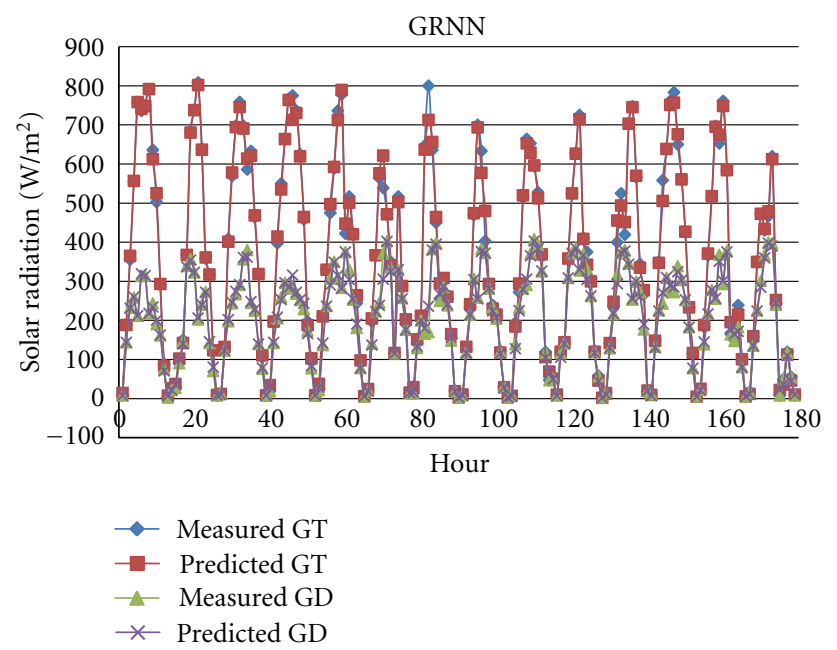

FIGURE 5: Solar radiation prediction using GRNN.

4.1. Solar Radiation Prediction Using GRNN. Figure 5 shows a sample of solar radiation prediction results using GRNN. The results are for June 2004.

In Figure 5, the prediction seems very accurate for both global and diffuse solar radiations. The evaluation of the network was performed using the proposed evaluation criteria. Table 1 shows the MABE, RMSE, and MBE for the selected months.

From the table, we can see that the prediction was accurate as shown by Figure 5 . The average MABE for both (global and diffuse) predictions was $4.9 \%$, and the average MRSE was 5.75\%, which means it was an efficient predictor. However, the average MBE was $0.29 \%$, which indicates a very slight underestimation by the predicted data. 
TABLE 1: Evaluation statistics for GRNN.

\begin{tabular}{|c|c|c|c|c|c|}
\hline Global radiation & MABE (\%) & $\operatorname{MRSE}\left(\mathrm{W} / \mathrm{m}^{2}\right)$ & MRSE (\%) & $\operatorname{MBE}\left(\mathrm{W} / \mathrm{m}^{2}\right)$ & MBE (\%) \\
\hline September 2000 & 4.5 & 25.9 & 5.7 & -1.5 & -0.33 \\
\hline March 2002 & 6.3 & 34.6 & 6.8 & -2.3 & -0.44 \\
\hline June 2004 & 3.5 & 19 & 4.5 & -2.8 & -0.07 \\
\hline Average & 4.8 & 26.5 & 5.7 & -2.2 & -0.28 \\
\hline \multicolumn{6}{|l|}{ Diffuse radiation } \\
\hline September 2000 & 4.7 & 15.8 & 5.7 & -1.5 & -0.33 \\
\hline March 2002 & 7 & 17.8 & 7.2 & -0.32 & -0.13 \\
\hline June 2004 & 3.3 & 11.4 & 4.5 & -1.11 & -0.44 \\
\hline Average & 5 & 15 & 5.8 & -0.98 & -0.3 \\
\hline
\end{tabular}

TABLE 2: Evaluation statistics for FFNN.

\begin{tabular}{|c|c|c|c|c|c|}
\hline Global radiation & MABE (\%) & $\operatorname{MRSE}\left(\mathrm{W} / \mathrm{m}^{2}\right)$ & MRSE (\%) & $\operatorname{MBE}\left(\mathrm{W} / \mathrm{m}^{2}\right)$ & MRSE (\%) \\
\hline September 2000 & 22.5 & 99.6 & 22.1 & -14.6 & -3.3 \\
\hline March 2002 & 20.2 & 101.1 & 19.9 & -7.6 & -1.5 \\
\hline June 2004 & 28.7 & 99 & 23.7 & 32.9 & 7.9 \\
\hline Average & 23.8 & 99.9 & 21.9 & 3.6 & 1.03 \\
\hline \multicolumn{6}{|l|}{ Diffuse radiation } \\
\hline September 2000 & 20.3 & 59.1 & 21.8 & -14.8 & -5.5 \\
\hline March 2002 & 32.2 & 61.8 & 25 & -7.6 & -3.1 \\
\hline June 2004 & 18.8 & 47.7 & 19 & 2.04 & .814 \\
\hline Average & 23.8 & 56.2 & 21.9 & -20.4 & -2.6 \\
\hline
\end{tabular}

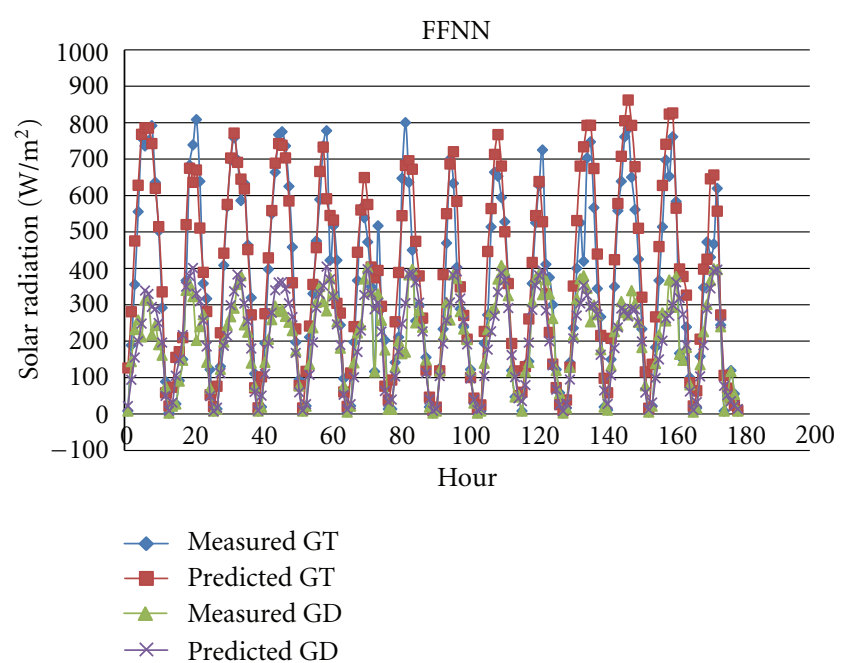

FIGURE 6: Solar radiation prediction using FFNN.

4.2. Solar Radiation Prediction Using FFNN. Figure 6 shows a sample of the prediction results using FFNN for June 2004. The results were good, but are not as accurate as the results from the GRNN.

Table 2 shows the evaluation of the solar radiation prediction using the FFNN. The results show that GRNN is clearly superior to FFNN. The average MABE, MRSE, and MBE were $23.8 \%, 21.9 \%$, and $-0.9 \%$, respectively. The results show a slight underestimation in the data, but on the other hand, there is a high MAPE, especially in the first and final hour of the solar day (from sunrise to sunset). Based on the results, $40 \%$ of the MAPE was caused by the first and last hours of the solar day. This shows that the GRNN is more efficient than the FFNN at predicting solar radiation in poor light conditions. In addition, the MRSE values show that the GRNN is more reliable than FFNN for future predictions as well.

4.3. Solar Radiation Prediction Using CFNN. Figure 7 shows a sample of the solar radiation prediction results using CFNN (June 2004). The CFNN prediction efficacy was close to the FFNN prediction efficacy. Both FFNN and CFNN yielded high prediction errors in the early and late periods of the solar day.

Table 3 shows the evaluation of the solar radiation prediction using CFNN. The CFNN prediction efficacy was quite close to the FFNN prediction efficacy with average MABE and MRSE values of $22.5 \%$ and $21.9 \%$, respectively. Using the CFNN to predict solar radiation has a higher percentage of underestimation than using the FFNN because the MBE percentage was $-19.15 \%$.

4.4. Solar Radiation Prediction Using ELMNN. Figure 8 shows a sample of solar radiation prediction using the ELMNN (June 2004). Based on these results, this prediction was the worst among all the proposed networks. 
TABLE 3: Evaluation statistics for CFNN.

\begin{tabular}{|c|c|c|c|c|c|}
\hline Global radiation & MABE (\%) & $\operatorname{MRSE}\left(\mathrm{W} / \mathrm{m}^{2}\right)$ & MRSE (\%) & $\operatorname{MBE}\left(\mathrm{W} / \mathrm{m}^{2}\right)$ & $\operatorname{MBE}(\%)$ \\
\hline September 2000 & 22.1 & 101.5 & 22.5 & -26.8 & -6 \\
\hline March 2002 & 21.8 & 108.2 & 21.3 & -23.12 & -4.6 \\
\hline June 2004 & 22.7 & 88.3 & 21.1 & 7.2 & 1.7 \\
\hline Average & 22.2 & 99.3 & 21.6 & -42.7 & -8.9 \\
\hline \multicolumn{6}{|l|}{ Diffused radiation } \\
\hline September 2000 & 20.6 & 59.3 & 21.9 & -13.6 & -5 \\
\hline March 2002 & 28.8 & 62.6 & 25.4 & -11.6 & -4.7 \\
\hline June 2004 & 18.9 & 48.1 & 19.2 & .86 & .34 \\
\hline Average & 22.8 & 56.7 & 22.2 & -24.3 & -9.4 \\
\hline
\end{tabular}

TABLe 4: Evaluation statistics for ELMNN.

\begin{tabular}{|c|c|c|c|c|c|}
\hline Global radiation & MABE (\%) & $\operatorname{MRSE}\left(\mathrm{W} / \mathrm{m}^{2}\right)$ & MRSE (\%) & $\operatorname{MBE}\left(\mathrm{W} / \mathrm{m}^{2}\right)$ & MRSE (\%) \\
\hline September 2000 & 28.1 & 131.9 & 29.3 & -34 & -8.9 \\
\hline March 2002 & 28.1 & 145.9 & 28.7 & -47.7 & -9.4 \\
\hline June 2004 & 33.4 & 128.8 & 30.8 & 9.9 & 2.4 \\
\hline Average & 29.9 & 135.5 & 29.6 & -23.9 & -15.9 \\
\hline Diffuse radiation & MABE (\%) & $\operatorname{RMSE}\left(\mathrm{W} / \mathrm{m}^{2}\right)$ & MRSE (\%) & $\operatorname{MBE}\left(\mathrm{W} / \mathrm{m}^{2}\right)$ & MRSE (\%) \\
\hline September 2000 & 41 & 126.8 & 46.8 & -34.7 & -12.8 \\
\hline March 2002 & 45.5 & 96.8 & 39.2 & -7.6 & -3.1 \\
\hline June 2004 & 31.1 & 78.6 & 31.3 & -7.4 & -3 \\
\hline Average & 39.2 & 100.7 & 39.1 & -16.6 & -6.3 \\
\hline
\end{tabular}

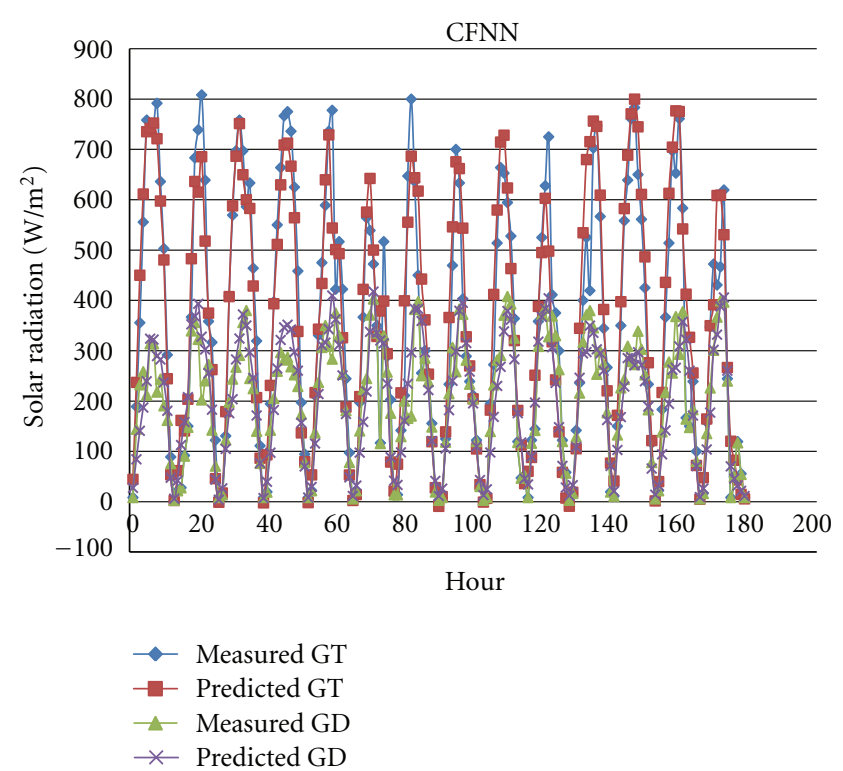

FIgURE 7: Solar radiation prediction results using CFNN.

Table 4 shows evaluation of the ELMNN for the solar radiation prediction. ELMN was the worst among the proposed networks since the average MABE, RMSE, and MBE values are $34.5 \%, 34.35 \%$, and $-11.1 \%$, respectively. Moreover, the ELMNN demonstrated an even worse diffuse solar radiation prediction compared to the global solar radiation prediction.

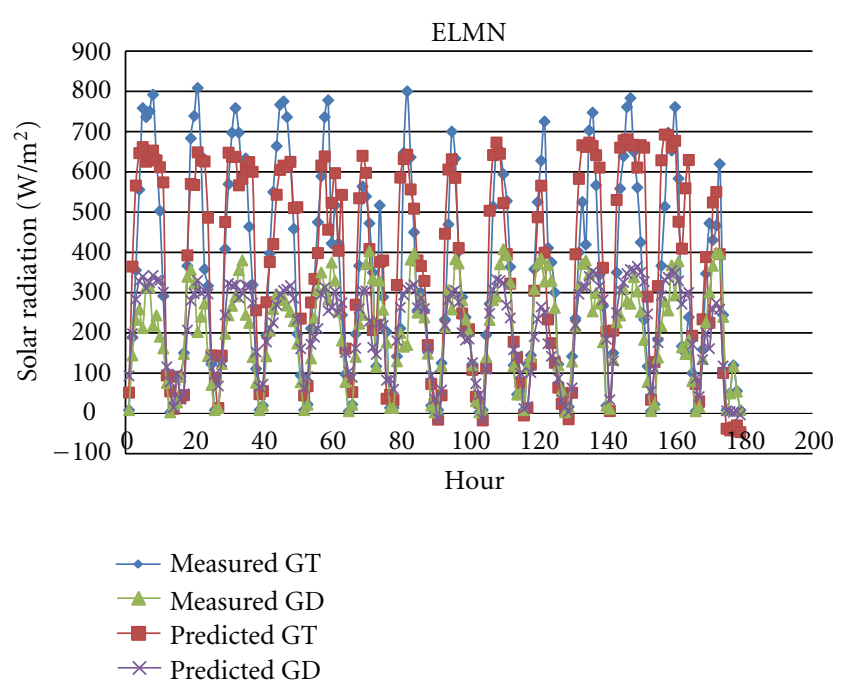

FIGURE 8: Solar radiation prediction results using ELMNN.

4.5. Proposed ANNs Comparison. Figures 9 and 10 show a comparison of measured and predicted values using the proposed ANNs. This comparison was based on monthly averages of global and diffuse solar radiations.

Based upon Figures 9 and 10, the GRNN exceeded the other methods with a very slight underestimation in global and diffuse solar radiation predictions. Meanwhile, the FFNN was superior to the CFNN in predicting diffuse solar radiation, while they both had similar efficacies in 


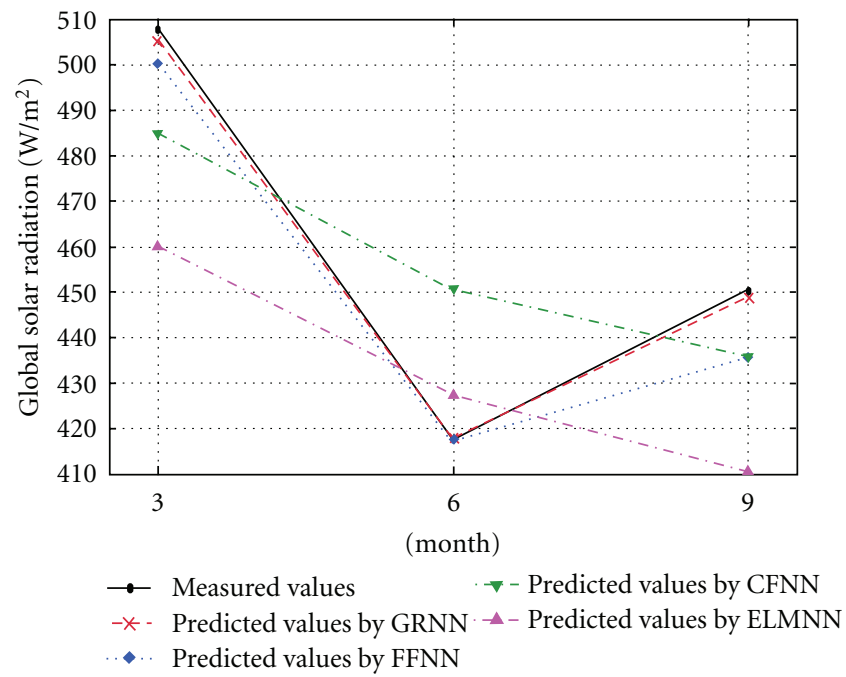

Figure 9: Comparison of measured and predicted values based on monthly averages of global radiation.

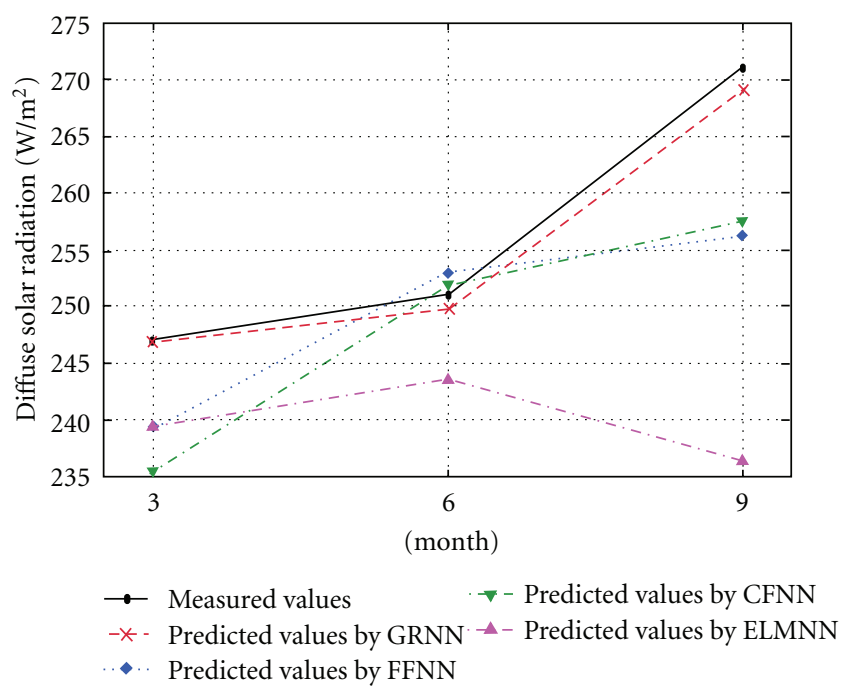

FIGURE 10: Comparison of measured and predicted values based on monthly averages of diffuse radiation.

TABLE 5: Comparison of the proposed methods.

\begin{tabular}{lccc}
\hline Network & MABE (\%) & MRSE (\%) & MBE (\%) \\
\hline GRNN & $4.9 \%$ & $5.75 \%$ & $0.29 \%$ \\
FFNN & $23 \%$ & $21.9 \%$ & $-0.9 \%$ \\
CFNN & $22.5 \%$ & $21.9 \%$ & $-19.15 \%$ \\
ELMNN & $34.5 \%$ & $34.35 \%$ & $-11.1 \%$ \\
\hline
\end{tabular}

predicting global solar radiation. The ELMNN was the worst among the proposed networks with a high underestimation in both global and diffuse solar radiation predictions. Table 5 shows a summary comparison of the proposed methods using the average percentage values of the MABE, MRSE, and MBE.

\section{Conclusion}

In this research, prediction of hourly solar radiation values for Kuala Lumpur was performed. This prediction was performed using the GRNN, FFNN, CFNN, and ELMNN artificial neural networks. Prediction results show that GRNN has a higher efficacy compared to the other proposed networks. The FFNN and CFNN are still efficient at predicting solar radiation but do not predict well in poor radiation conditions such as the first and final hour of the solar day. The ELMNN was the worst at predicting the solar radiation among the proposed methods. Based on our results, GRNN is recommended for such purposes in Malaysia and other nearby regions.

\section{References}

[1] J. A. Duffie and W. A. Beckman, Solar Engineering of Thermal Processes, John Wiley \& Sons, New York, NY, USA, 1991.

[2] S. Alam, S. C. Kaushik, and S. N. Garg, "Computation of beam solar radiation at normal incidence using artificial neural network," Renewable Energy, vol. 31, no. 10, pp. 1483-1491, 2006.

[3] S. M. Al-Alawi and H. A. Al-Hinai, "An ANN-based approach for predicting global radiation in locations with no direct measurement instrumentation," Renewable Energy, vol. 14, no. 14, pp. 199-204, 1998.

[4] H. K. Elminir, Y. A. Azzam, and F. I. Younes, "Prediction of hourly and daily diffuse fraction using neural network, as compared to linear regression models," Energy, vol. 32, no. 8, pp. 1513-1523, 2007.

[5] L. Hontoria, J. Aguilera, and P. Zufiria, "Generation of hourly irradiation synthetic series using the neural network multilayer perceptron," Solar Energy, vol. 72, no. 5, pp. 441-446, 2002.

[6] K. Mehrotra, C. K. Mohan, and S. Ranka, Elements of Artificial Neural Networks, MIT Press, 1996.

[7] A. Mellit, M. Benghanem, and M. Bendekhis, "Artificial neural network model for prediction solar radiation data: application for sizing stand-alone photovoltaic power system," in Proceedings of the IEEE Power Engineering Society General Meeting, vol. 1, pp. 40-44, June 2005.

[8] G. Mihalakakou, M. Santamouris, and D. N. Asimakopoulos, "The total solar radiation time series simulation in Athens, using neural networks," Theoretical and Applied Climatology, vol. 66, no. 3-4, pp. 185-197, 2000.

[9] M. Mohandes, S. Rehman, and T. O. Halawani, "Estimation of global solar radiation using artificial neural networks," Renewable Energy, vol. 14, no. 1-4, pp. 179-184, 1998.

[10] A. Sözen, E. Arcaklioğlu, M. Özalp, and N. Çağlar, "Forecasting based on neural network approach of solar potential in Turkey," Renewable Energy, vol. 30, no. 7, pp. 1075-1090, 2005.

[11] D. G. S. Chuah and S. L. Lee, "Solar radiation in peninsular Malaysia-Statistical presentations," Energy Conversion and Management, vol. 22, no. 1, pp. 71-84, 1982.

[12] K. Sopian and M. Y. H. Othman, "Estimates of monthly average daily global solar radiation in Malaysia," Renewable Energy, vol. 2, no. 3, pp. 319-325, 1992.

[13] M. Caudill and C. Butler, Understanding Neural Networks: Computer Explorations Volume 1: Basic Networks, The MIT Press, Cambridge, Mass, USA, 1993.

[14] H. Cihan, Intelligent Engineering Systems Through Artificial Neural Network, ASME, 2000. 


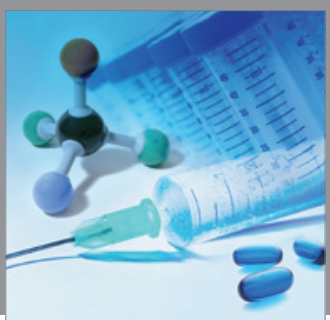

International Journal of

Medicinal Chemistry

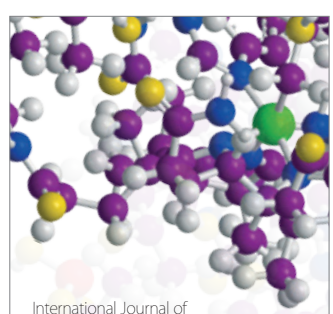

Carbohydrate Chemistry

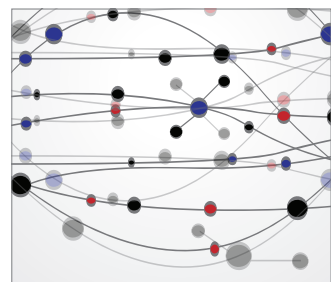

The Scientific World Journal
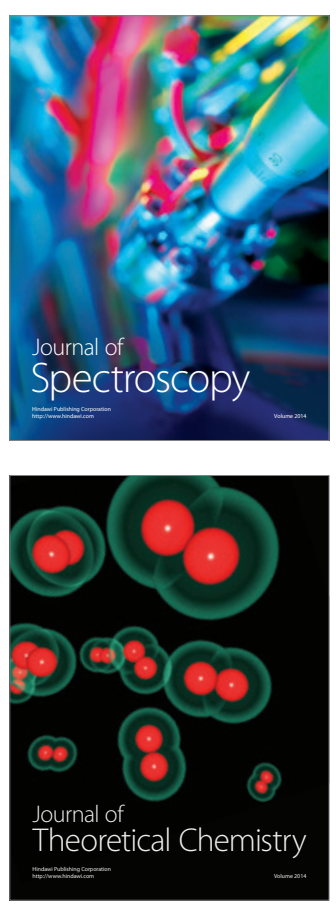
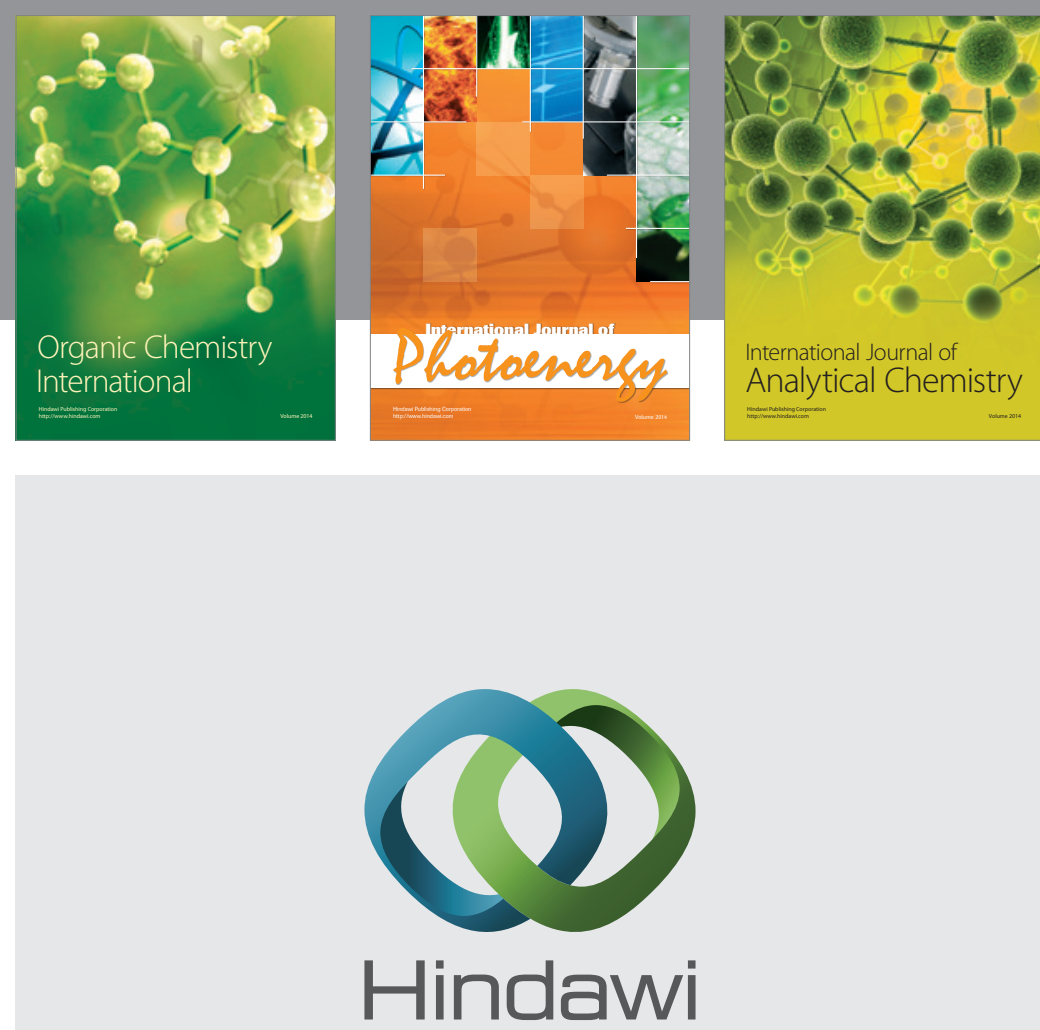

Submit your manuscripts at

http://www.hindawi.com
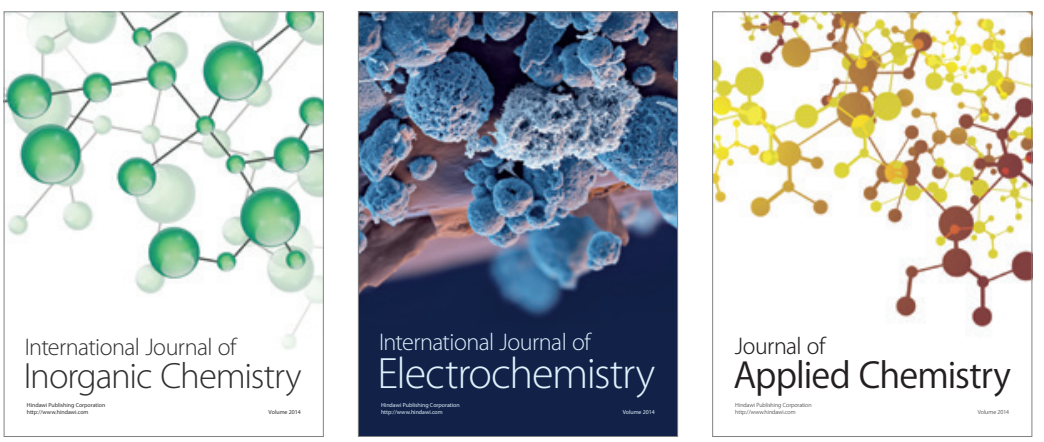

Journal of

Applied Chemistry
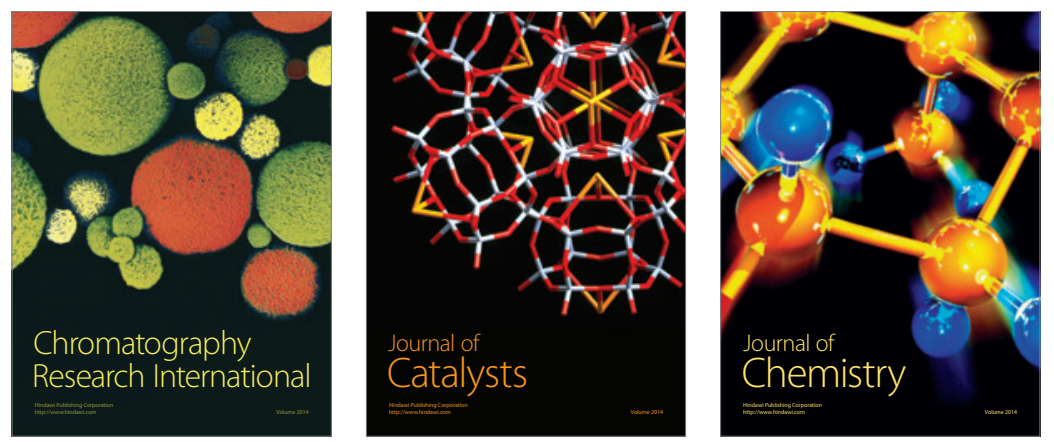
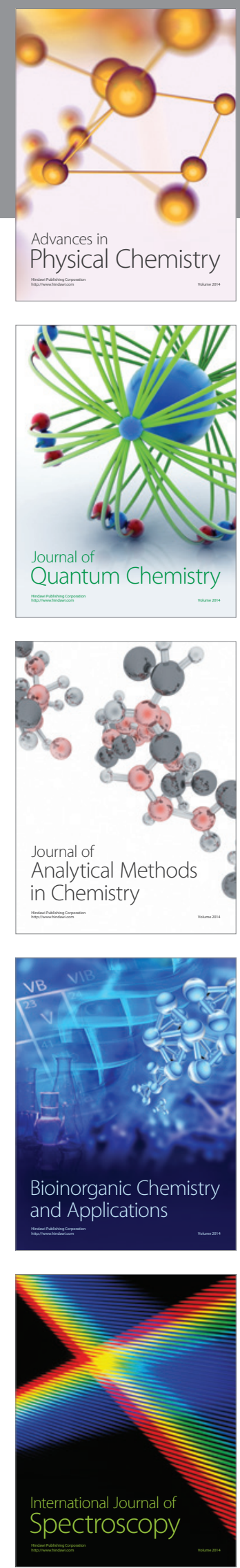